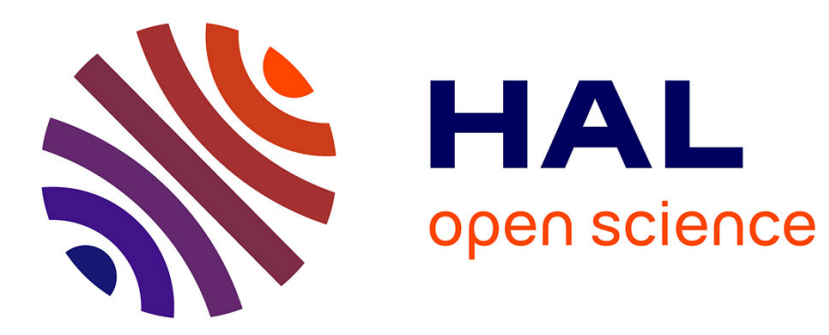

\title{
Three-point bending test of pantographic blocks: numerical and experimental investigation
}

\author{
M. Erden Yildizdag, Emilio Barchiesi, Francesco Dell'Isola
}

\section{To cite this version:}

M. Erden Yildizdag, Emilio Barchiesi, Francesco Dell'Isola. Three-point bending test of pantographic blocks: numerical and experimental investigation. Mathematics and Mechanics of Solids, 2020, 14 p. hal-02914321

\section{HAL Id: hal-02914321 \\ https://hal.science/hal-02914321}

Submitted on 11 Aug 2020

HAL is a multi-disciplinary open access archive for the deposit and dissemination of scientific research documents, whether they are published or not. The documents may come from teaching and research institutions in France or abroad, or from public or private research centers.
L'archive ouverte pluridisciplinaire HAL, est destinée au dépôt et à la diffusion de documents scientifiques de niveau recherche, publiés ou non, émanant des établissements d'enseignement et de recherche français ou étrangers, des laboratoires publics ou privés. 


\title{
Three-point bending test of pantographic blocks: numerical and experimental investigation
}

M Erden Yildizdag

Department of Naval Architecture and Ocean Engineering, Istanbul Technical University, Maslak, Istanbul, Turkey; International Research Center for the Mathematics and Mechanics of Complex Systems, University of L’Aquila, Italy

\section{Emilio Barchiesi}

International Research Center for the Mathematics and Mechanics of Complex Systems, University of L’Aquila, Italy

\section{Francesco dell'Isola}

International Research Center for the Mathematics and Mechanics of Complex Systems, University of L'Aquila, Italy;

Dipartimento di Ingegneria Civile, Edile-Architettura e Ambientale, Universitá degli Studi dell'Aquila, L'Aquila, Italy

\begin{abstract}
The equilibrium forms of pantographic blocks in a three-point bending test are investigated via both experiments and numerical simulations. In the computational part, the corresponding minimization problem is solved with a deformation energy derived by homogenization within a class of admissible solutions. To evaluate the numerical simulations, series of measurements have been carried out with a suitable experimental setup guided by the acquired theoretical knowledge. The observed experimental issues have been resolved to give a robust comparison between the numerical and experimental results. Promising agreement between theoretical predictions and experimental results is demonstrated for the planar deformation of pantographic blocks.
\end{abstract}

\section{Keywords}

Mechanical metamaterials, pantographic structures, three-point bending, second gradient modeling, additive manufacturing 


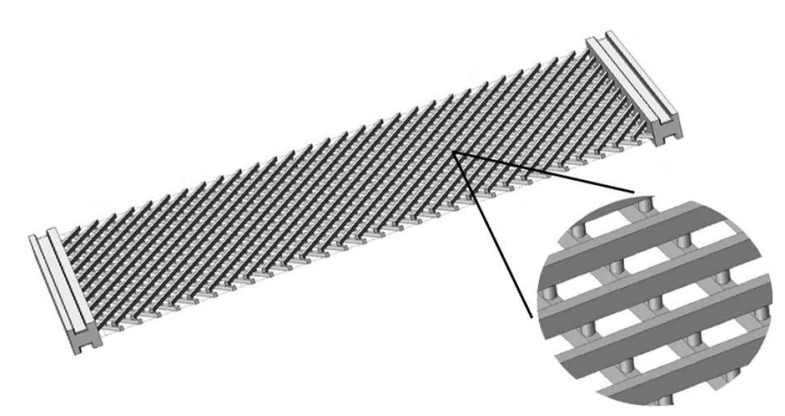

Figure I. Topology of pantographic sheets.

\section{Introduction}

In the last decade, with the impressive progress in advanced manufacturing techniques (e.g. 3D printing), the concept of mechanical metamaterials has become a popular topic, owing to their promising features in different engineering applications (for example, see Kadic et al. [1], Lee et al. [2], Turco et al. [3], Vangelatos et al. [4-6], Eugster et al. [7], and Nejadsadeghi et al. [8]). Mechanical metamaterials are multiscale structures whose overall mechanical response is determined by the interactions between structural constituents at different length scales. By controlling structural patterns at different scales, one can design and fabricate metamaterials with exotic and unnatural properties, such as high stiffness-to-weight ratio, high toughness, and negative Poisson ratio. We refer the reader to the extensive review papers of Barchiesi et al. [9] and Yu et al. [10] for the state of the art in the study of mechanical metamaterials.

In this study, we particularly focus on a type of mechanical metamaterial, namely, pantographic blocks. This kind of mechanical metamaterial consists of layered pantographic sheets that contain two families of intersecting fibers connected with pivots (see Figure 1). Pantographic sheets have been extensively investigated recently (for example, see dell'Isola et al. [11, 12]). Actually, pantographic sheets are one of the different versions of pantographic microstructures that have been introduced as an example of second gradient continuum, i.e. a continuum whose deformation energy depends on the second gradient of displacement (see Alibert et al. [13]). The central theme of this type of continuum was first introduced by Gabrio Piola in the period 18451850 (see dell'Isola et al. [14-16], Auffray et al. [17] and Rahali et al. [18]) as a result of his forerunning micro-to-macro identification procedure based on the postulation of the validity of a discrete Lagrangian model at microscale and a continuous model whose kinematics is given by a displacement field at macroscale. The existence of a continuum whose deformation energy depends on the second gradient of displacement has been investigated in many studies. For example, see Pideri and Seppecher [19], Abdoul-Anziz and Seppecher [20], and Abdoul-Anziz et al. [21].

In the studies presented up to now, most of the investigations are limited to planar deformations of 2D continuum models for pantographic sheets. Dell'Isola et al. [22] presented a second gradient model for the planar deformation of pantographic sheets. Cuomo et al. [23] and dell'Isola et al. [24] analyzed the behavior of pantographic sheets under bias-extension test. Placidi et al. [25] demonstrated a 2D second gradient model for pantographic sheets formed with inextensible fibers. Spagnuolo et al. [26] investigated pivot damage issues occurring in aluminum pantographic sheets. Andreaus et al. [27] presented a finite-element method framework for the static analysis of pantographic structures modeled using non-linear Euler-Bernoulli beams. De Angelo et al. [28] investigated the behavior of metallic pantographic sheets. In a number of papers, researchers have worked on the identification of constitutive parameters for second gradient materials (see Giorgio [29], Placidi et al. [30], Yang et al. [31], De Angelo et al. [32], Nejadsadeghi et al. [33], Solyaev et al. [34], and Turco [35]). In a few studies, the $3 \mathrm{D}$ deformation of pantographic structures has been investigated in the context of second gradient modeling, for example in the studies presented by Steigmann and dell'Isola [36], Giorgio et al. [37, 38], and Scerrato et al. [39]. Additionally, "higher grade" (see Niiranen and Niemi [40] for plates, and Khakalo et al. [41], Khakalo and Niiranen [42], and Barchiesi and Khakalo [43] for lattice structures) and "higher order" (see Eremeyev [44], Eremeyev et al. [45], Eremeyev and Altenbach [46], Altenbach and Eremeyev [47-49], and Altenbach et al. [50]) models have been popularly proposed to predict the behavior of micro-architectured metamaterials. The importance of such models has been discovered for different applications and phenomena. For interested readers, we refer to the recently published works of Misra and Poorsolhjouy [51], Placidi et al. 


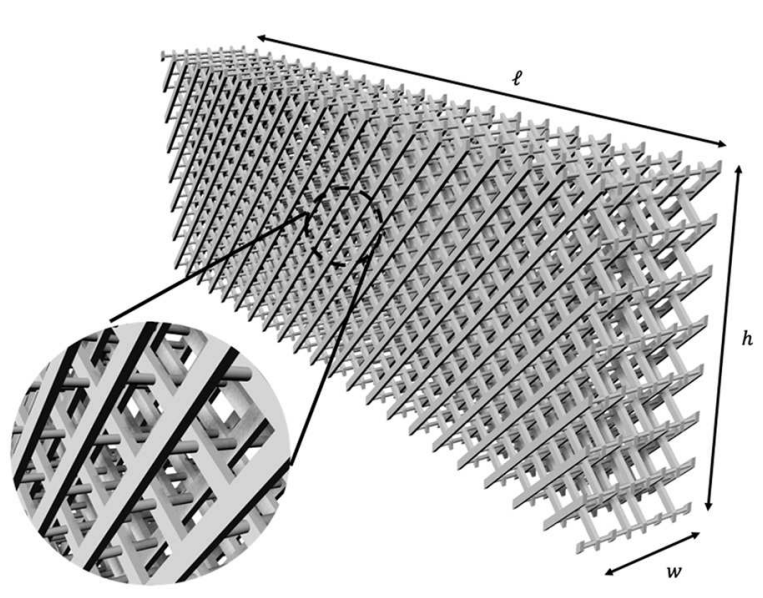

Figure 2. Design of a pantographic block.

[52], Turco et al. [53-56], Battista et al. [57], Greco et al. [58] Franciosi et al. [59], Chiaia et al. [60], Barchiesi et al. [61], and Spagnuolo and Andreaus [62] for further details.

The interest in pantographic microstructures has been motivated to prove that second and higher gradient models are indeed conceivable and realizable, thus not being meaningless mathematical abstractions, and, therefore, the ideas inspired by Piola [14], Green [63], Mindlin [64], Sedov [65], Toupin [66], and Germain [67] can be fruitfully exploited for applications of non-classical continuum modeling. It is also worth remarking that an interesting set of exotic effects and phenomena [68-71], which may lead to promising technological applications, has been observed (see dell'Isola et al. [72] and della Corte et al. [73]).

In regard to previously considered research efforts and the latest advancements in additive manufacturing technologies, it seems natural to try to conceive, design, fabricate, and test some truly 3D mechanical metamaterials involving pantographic microstructures. Therefore, in this study, pantographic blocks under three-point bending tests are studied. According to the authors' best knowledge, this is the first study to investigate threepoint bending tests of pantographic blocks, both numerically and experimentally. For this purpose, the first step is to form a design that has superimposed pantographic layers with elastic pivots, as given in Figure 2. Then, the standard three-point bending test is performed for fabricated pantographic blocks, and a comparison between numerical and experimental results is made to show the validity of the mathematical model. In this study, the designed specimens have been fabricated with a 3D printing technology, namely, selective laser sintering. Here, we are aware of the limits of our modeling and numerical analysis: we assume that the planar model can be used by modifying some constitutive parameters accordingly. This courageous assumption has been proven to have some ground, rather than satisfactory coincidence obtained with the experimental evidence, as will be discussed in the next sections.

The organization of the rest of the paper is as follows. In Section 2, the mathematical model adopted for the numerical simulations is briefly summarized. Next, the experimental setup and related issues are explained in Section 3. In Section 4, the numerical results are presented for the pantographic block under study, and the agreement with experimental results is shown. Finally, in Section 5, we highlight our conclusions and try to provide some insights for future studies based on our observations.

\section{Mathematical model: macro-level second gradient model for planar pantographic structures}

In this section, the mathematical model adopted for the analysis of pantographic block under study is presented. As mentioned earlier, it is assumed that the considered pantographic block has planar deformation; therefore, the mathematical model given by dell'Isola et al. [22] for pantographic sheets is adopted in the simulations. In the framework presented by dell'Isola et al. [22], the macro-level model was formulated with a heuristic micro-macro identification procedure which was first pioneered by Gabrio Piola [14]. In this context, to model pantographic sheets, a discrete model is introduced for the microstructured 2D continuum consisting of two families of orthogonal fibers in the reference configuration. The discrete model contains a set of particles connected to each other by appropriate extensional and rotational springs in a $2 \mathrm{D}$ space. As can be seen from 


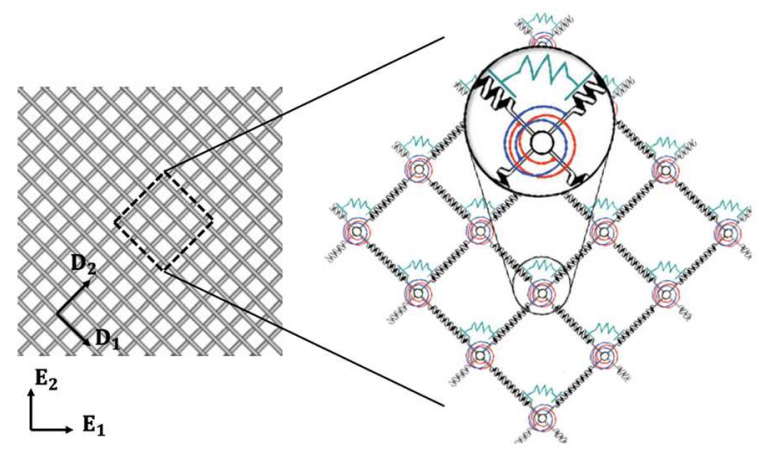

Figure 3. Discrete model for pantographic sheets.

Figure 3, particles are located at the intersections of fibers and are connected to each other by extensional springs. An additional three rotational springs are introduced to model the behavior of the structure due to pivots at intersections.

By following the heuristic homogenization procedure, the deformation energies at macroscale are identified by using the postulated deformation energies for the discrete model at the microscale. Therefore, the macroscopic deformation energies (i.e. the macroscopic Lagrangian density functionals) are defined by means of microscopic constitutive parameters.

To introduce the kinematics, the current position of a material point $(\mathbf{x})$ is written as

$$
\mathbf{x}=\chi(\mathbf{X})=\left(X^{\alpha}+u^{\alpha}\left(X^{\beta}\right)\right) \mathbf{D}_{\alpha},
$$

where $\mathbf{D}_{\alpha}$ is the Lagrangian basis directed along the two families of fibers, as given in Figure 3, with $\alpha, \beta=1,2$. Also, $u^{\alpha}$ represents the components of the displacement field, and $\left\{E_{1}, E_{2}\right\}$ is the fixed Cartesian basis.

Here, the current configuration is defined by the following map:

$$
\chi=\Omega \rightarrow \mathcal{R}^{2}
$$

where $\Omega$ is a rectangular domain. The unit vectors tangent to the fibers in the current configuration are obtained by

$$
\mathbf{e}_{\alpha}=\frac{\mathbf{F D}_{\alpha}}{\left\|\mathbf{F D}_{\alpha}\right\|},
$$

where $\mathbf{F}$ is the deformation gradient, $\mathbf{F}=\nabla \chi$. In the model, a strain energy density, which includes stretching, bending, and shearing effects (see dell'Isola et al. [22] for further details), is introduced, as follows:

$$
W\left(\varepsilon_{\alpha}, \kappa_{\alpha}, \gamma\right)=\sum_{\alpha}\left(\frac{1}{2} K_{\mathrm{e}} \varepsilon_{\alpha}^{2}+\frac{1}{2} K_{\mathrm{b}} \kappa_{\alpha}^{2}\right)+\frac{1}{2} K_{\mathrm{p}} \gamma^{2},
$$

where $\varepsilon_{\alpha}$ is the stretch of fibers,

$$
\varepsilon_{\alpha}=\left\|\mathbf{F D}_{\alpha}\right\|-1
$$

$\kappa_{\alpha}$ is the fiber curvature,

$$
\kappa_{\alpha}=\left\|\mathbf{c}_{\alpha}-\left(\mathbf{c}_{\alpha} \cdot \mathbf{c}_{\alpha}\right) \mathbf{e}_{\alpha}\right\|=\left\|\left(\mathbf{I}-\mathbf{e}_{\alpha} \otimes \mathbf{e}_{\alpha}\right) \mathbf{c}_{\alpha}\right\|,
$$

and $\gamma$ denotes the shear distortion related to the angle change between fibers,

$$
\gamma=\left|\cos ^{-1}\left(\mathbf{e}_{1} \cdot \mathbf{e}_{2}\right)-\frac{\pi}{2}\right| .
$$




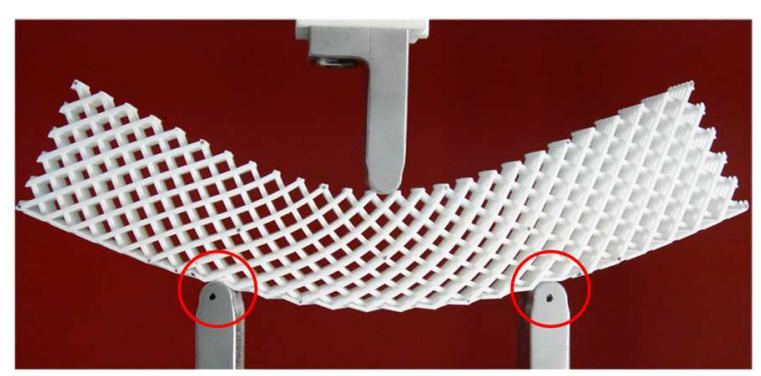

Figure 4. Cell trapping during three-point bending test of a pantographic block.

Here, $\mathbf{c}_{\alpha}$ is an auxiliary vector field given by

$$
\mathbf{c}_{\alpha}=\frac{\nabla \mathbf{F} \mid \mathbf{D}_{\alpha} \otimes \mathbf{D}_{\alpha}}{\left\|\mathbf{F} \mathbf{D}_{\alpha}\right\|}
$$

where $\left(\nabla \mathbf{F} \mid \mathbf{D}_{\alpha} \otimes \mathbf{D}_{\alpha}\right)^{\beta}=\partial_{\alpha} F_{\alpha}^{\beta}=\partial_{\alpha \alpha} \chi^{\beta}$. The terms $K_{\mathrm{e}}, K_{\mathrm{b}}$, and $K_{\mathrm{p}}$ are the constant and positive material parameters related to stretching, bending, and shearing, respectively.

Finally, the governing equations are obtained with the variational statement

$$
\delta \int_{\Omega} W\left(\varepsilon_{\alpha}, \kappa_{\alpha}, \gamma\right) \mathrm{d} \Omega=0 \quad \forall \delta \mathbf{u}
$$

where $\delta \mathbf{u}$ belongs to the vector space of admissible displacement variations, i.e. test functions. In this study, since quasistatic deformation is considered, kinetic energy is not included in the expression given in equation (9). Each obtained shape that corresponds to an imposed displacement boundary condition is the corresponding equilibrium configuration, i.e. the shape that minimizes the deformation energy. The used numerical code has been implemented with the standard packages available in COMSOL Multiphysics ${ }^{\circledR}$, namely, Structural Mechanics and Weak Form PDE. The boundary conditions at contact points (i.e. free sliding with no penetration between specimen and blades) are applied using the Structural Mechanics package. Moreover, aimed at mixed finite-element formulations, an extra kinematic tensorial descriptor is introduced in the Weak Form PDE package to be able to compute the terms related to the second gradient of the displacement field. This extra kinematic descriptor is constrained to be equal to the gradient of displacement field.

We are aware of the fact that such a numerical code can be optimized for lower computational burdens, for instance, by using an isogeometric approach (as those presented in Fischer et al. [74], Cuomo et al. [75], Cazzani et al. [76-79], Niiranen et al. [80-82], Balobanov and Niiranen [83], Khakalo and Niiranen [84, 85], Yaghoubi et al. [86], Yildizdag et al. [87, 88], Capobianco et al.[89], Greco et al. [90], and Balobanov et al. [91]).

\section{Experimental apparatus and measurements}

In this section, we detail our designed experimental setup and conducted measurements. Pantographic blocks of length $\ell$, height $h$, and width $w$ are considered in this study. The designed pantographic blocks consist of fibers with rectangular cross-section, and they have been fabricated using selective laser sintering technology for experiments (see computer-aided design shown in Figure 2). Each designed pantographic block has a microstructure characterized by the following parameters: the distance between two subsequent pivots, which is referred to as cell dimension, $\ell_{\mathrm{p}}$, pivot radius, $r_{\mathrm{p}}$, and the rectangular cross-section of fibers with height $a$ and width $b$.

To get reliable experimental results, owing to the unique structural pattern of the specimen under study, some simple modifications need to be made to conduct three-point bending tests of pantographic blocks. The standard blades used in three-point bending tests have a small radius of curvature. This situation leads to an important issue in the three-point bending test of pantographic blocks. The experiments conducted with different pantographic blocks show that specimens may get "trapped" in blades during the test, and, therefore, cells trapped in blades may have excessive deformation, as shown in Figure 4. In this case, it is clear that the continuum model used for numerical simulations is not appropriate to describe the target physical situation. 

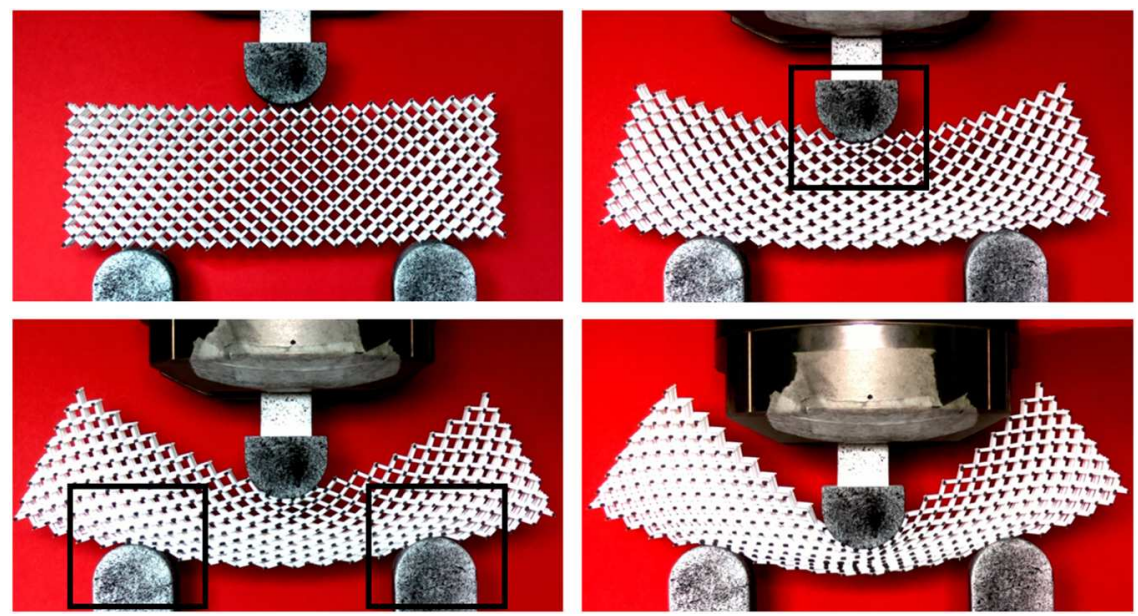

Figure 5. Excessive deformation of cells in contact with larger blades during three-point bending test.

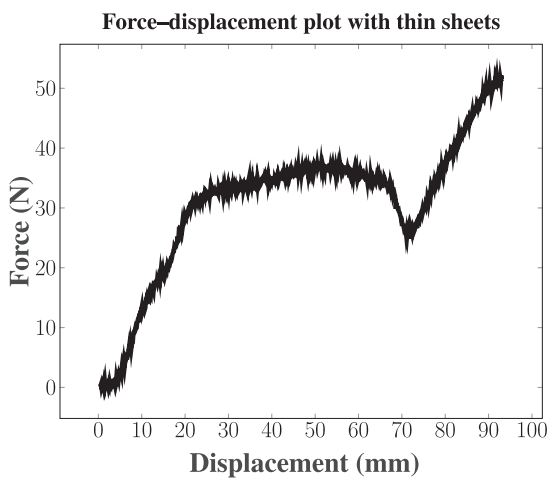

(a)

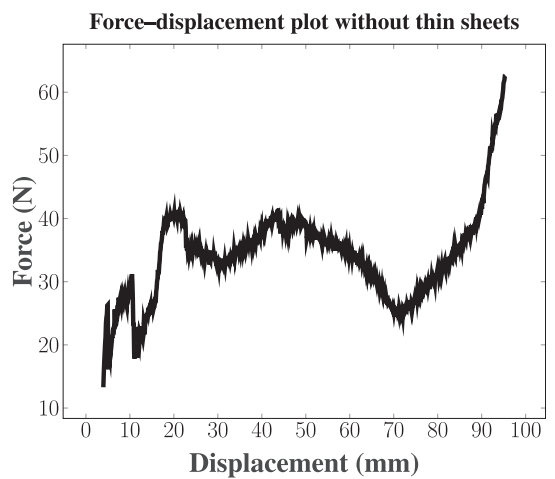

(b)

Figure 6. Force-displacement plots with and without thin sheets between pantographic block and blades.

To resolve this issue, blades with relatively larger radius of curvature have been designed for experiments (see Figure 5). However, even with larger blades, it is observed that sliding of specimens is not uniform, and some pantographic cells in contact with blades have local deformation patterns, as can be seen from Figure 5. As a result, a "jumping" pattern in the experimental plot (imposed displacement as a function of exerted total reactive force) is obtained (see Figure 6(b)).

The experimental solution used in this study is simple and effective. As can be seen from Figure 7, at the beginning of the process, very light and flexible thin sheets are placed between pantographic blocks and the blades. The thin layer is chosen in such a way that it adheres perfectly to the lower pivots of the pantographic block and slides perfectly on the blades. This solution has been proven to be much more efficient compared to other solutions which were tried earlier. In Figure 8, the behavior of a pantographic block under three-point bending test is presented. In this case, it is clear that the thin layers prevent having undesired behavior around the blades. Furthermore, In Figure 6, the force-displacement plots obtained by conducted experiments with and without thin sheets are compared to show the validity of our practical experimental solution. As can be seen from Figure 6(b), in case the three-point bending test is conducted without thin sheets, the force-displacement plot have some certain jumps because of excessive deformation of the cells in contact with blades, and it is also observed that the pantographic block slides instantly during the experiment. On the other hand, as seen from Figure 6(a), due to the thin sheets placed between the pantographic block and blades, a reliable forcedisplacement relation is obtained in the experiment. Therefore, this practical approach allows us to provide a better comparison between the numerical and experimental results in the following section.

In three-point bending tests, the device imposes the given displacements, and the reactive forces are measured for each corresponding imposed displacement. Here, the displacement of central blade is applied slow enough that the observed deformation pattern can be regarded as quasistatic, and therefore, it is assumed that 


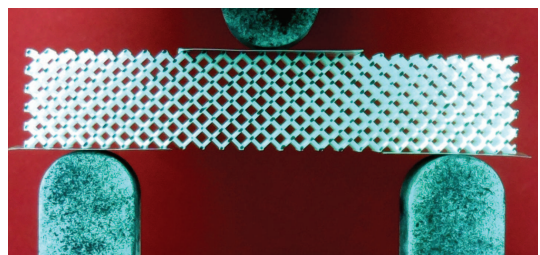

(a) Experiment $\left(u_{0}=0 \mathrm{~m}\right)$.

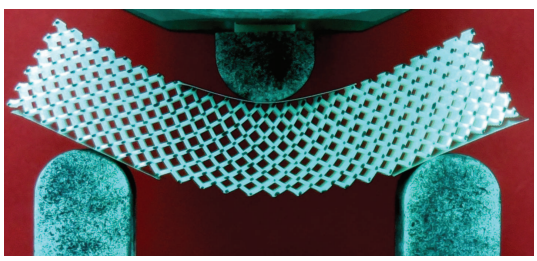

(c) Experiment $\left(u_{0}=20 \mathrm{~mm}\right)$.

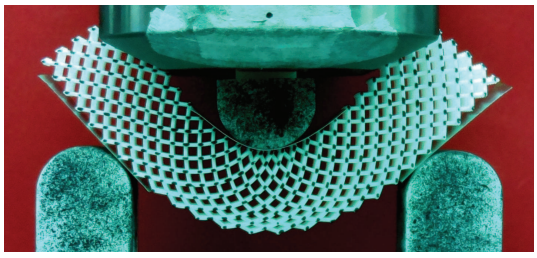

(e) Experiment $\left(u_{0}=40 \mathrm{~mm}\right)$.

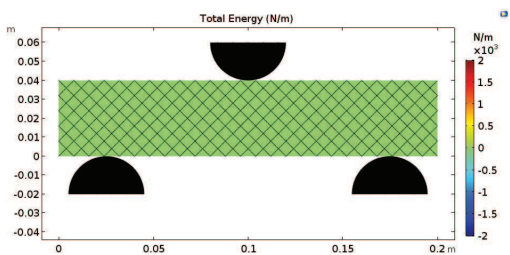

(b) Simulation $\left(u_{0}=0 \mathrm{~mm}\right)$.

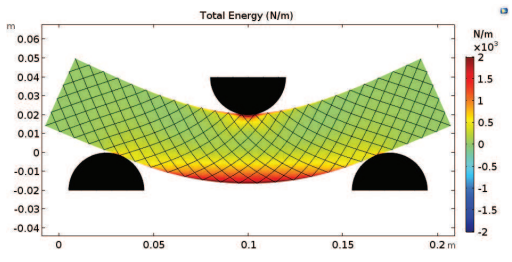

(d) Simulation $\left(u_{0}=20 \mathrm{~mm}\right)$.

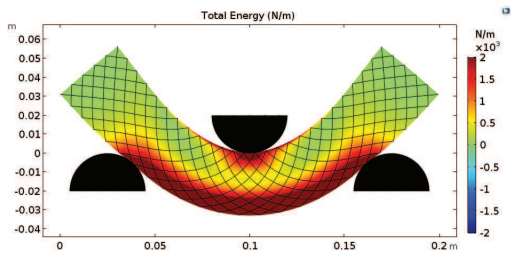

(f) Simulation $\left(u_{0}=40 \mathrm{~mm}\right)$.

Figure 9. Comparison of experimental and numerical results.

numerical simulations, the prescribed displacement boundary condition is imposed at the tip of the top blade to simulate the three-point bending test, while the other blades are kept fixed. The fitting process for constitutive parameters followed the micro-macro identification presented by Giorgio [29]. As a result of this identification procedure, the following stiffness parameters are used in the numerical simulations: $K_{\mathrm{e}}=4.75 \times 10^{6} \mathrm{~N} / \mathrm{m}$, $K_{\mathrm{b}}=2.79 \times 10^{-1} \mathrm{~N} \mathrm{~m}$, and $K_{\mathrm{p}}=5.95 \times 10^{4} \mathrm{~N} / \mathrm{m}$.

In Figure 9, the experimental and numerical results are compared when the displacement of top blade $\left(u_{0}\right)$ is equal to 0,20, and $40 \mathrm{~mm}$, respectively. In the numerical results given in Figure 9(b), (d), and (f), the total energy $(W)$ of the structure is plotted using COMSOL Multiphysics ${ }^{\circledR}$.

As can be seen from Figure 9, the presented numerical results compare very well with those obtained by experiments. The behavior of the pantographic block is quite similar in both the numerical simulation and experiment: it is clear that similar equilibrium shapes are observed in both numerical and experimental results. To have a better comparison, material lines oriented as fibers are assigned in the second gradient continua to compare their behavior with the deformation of fibers in the experiment. In Figure 10, deformed shapes of fibers and material lines are compared when the displacement of the top blade is equal to $40 \mathrm{~mm}$. It is clear that the deformed configurations of both fibers and material lines look very similar. Importantly, it is also observed that the tips of the pantographic block expand in the direction of height with increasing values of imposed displacement. This behavior is observed because of the contraction in the center of the pantographic block during the experiment.

Moreover, we investigated the contribution of each term appearing in the deformation energy given in equation (4). In the mathematical model, the total energy has three main parts,

$$
W=W_{\mathrm{e}}+W_{\mathrm{b}}+W_{\mathrm{s}},
$$

where $W_{\mathrm{e}}, W_{\mathrm{b}}$, and $W_{\mathrm{s}}$ are extensional, bending, and shearing energies, respectively. The contribution of each term is plotted in Figure 11(a) to (c), respectively, for extensional, bending, and shearing energies, when the displacement of the top blade is equal to $40 \mathrm{~mm}$. The extensional energy plotted in Figure 11(a) shows that the energy due to extension is slightly high in the top and bottom surfaces of the pantographic block. Conversely, as can be seen from Figure 11(b), the bending energy is higher around the middle of the pantographic block. Here, an interesting result has been observed in Figure 11(c) for shearing energy: shearing due to the existence of pivots plays an important role in the behavior of the pantographic block. The shearing energy is relatively 


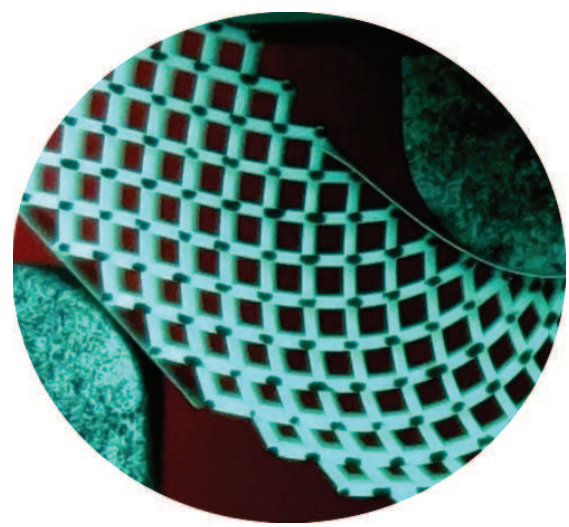

(a) Experiment $\left(u_{0}=40 \mathrm{~mm}\right)$.

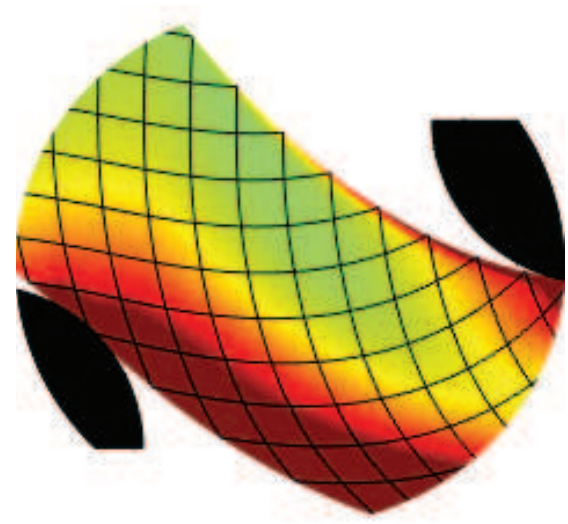

(b) Simulation $\left(u_{0}=40 \mathrm{~mm}\right)$.

Figure 10. Behavior of fibers in experiment compared with material lines in the simulation.

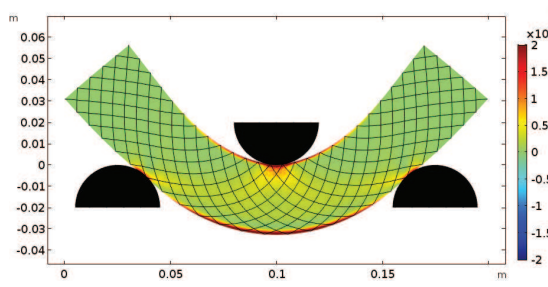

(a) Extensional energy $\left(W_{\mathrm{e}}\right)$.

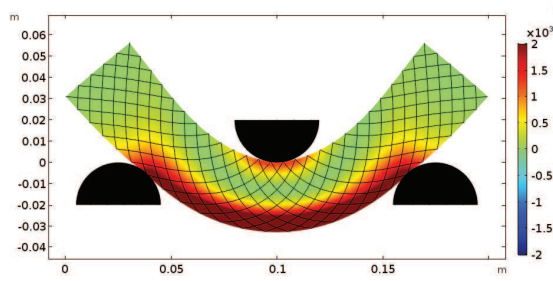

(c) Shearing energy $\left(W_{\mathrm{s}}\right)$.

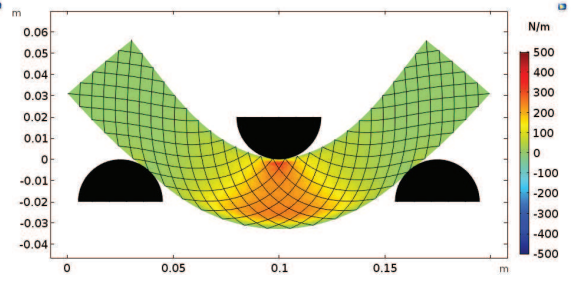

(b) Bending energy $\left(W_{\mathrm{b}}\right)$.

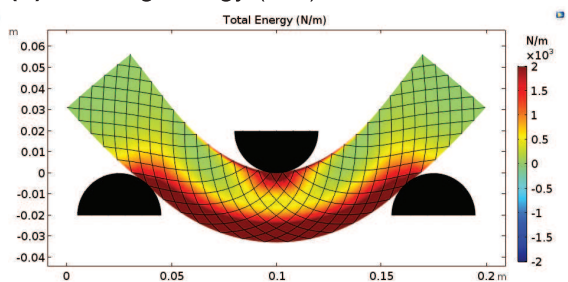

(d) Total energy $(W)$.

Figure I I. Contribution of each energy term.

larger compared with the other components of total energy, and it is significantly high in the lower part of the pantographic block. Therefore, it can be concluded that most of the total energy is dependent on shearing effects.

Additionally, the same problem is solved in the context of classical first gradient theory (i.e. Cauchy continuum) and the results are compared with those obtained using the proposed second gradient model. In Figure 12 , numerical results with the first and second gradient models are presented when the displacement of the top blade $\left(u_{0}\right)$ is equal to 0,20 , and $40 \mathrm{~mm}$, respectively. In each case given in Figure 12, the total energy $(W)$ of the system is plotted using COMSOL Multiphysics ${ }^{\circledR}$. As can be seen from Figures 12(e) and (f), a significant difference between the first and second gradient models is observed. In the first gradient model, the total energy is significantly concentrated in the upper and lower parts of the structure (Figure 12(e)). However, in the second gradient model, the total energy spreads out, especially along the lower part of the pantographic block (Figure 12(f)). In addition, the final equilibrium shape of the structure obtained with the first gradient model is considerably different in comparison with the equilibrium shapes obtained by experiments. Therefore, it can be concluded that the classical first gradient model produces an inadequate result. For those who want to use the first gradient model, the best way is to model the system with appropriate beam elements (or maybe solid elements), and then conduct the finite-element modeling analysis by applying suitable conditions at each pivot!

Finally, the force-displacement curves obtained via both the experiment and simulations (both second gradient and first gradient) are compared in Figure 13. Here, in the numerical simulation, the force plotted in Figure 13 is the computed reaction force at the top blade. The comparison clearly shows that the simulation 


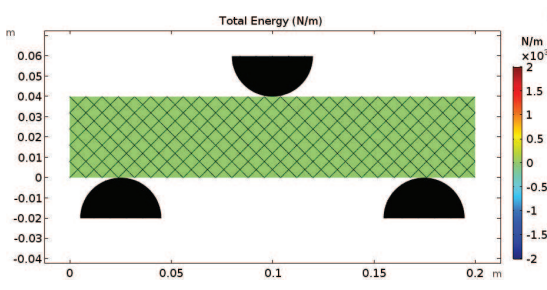

(a) First gradient model $\left(u_{0}=0 \mathrm{~mm}\right)$.

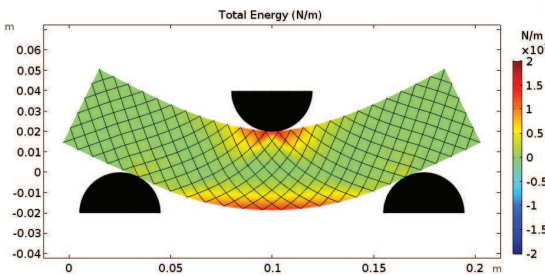

(c) First gradient model $\left(u_{0}=20 \mathrm{~mm}\right)$.

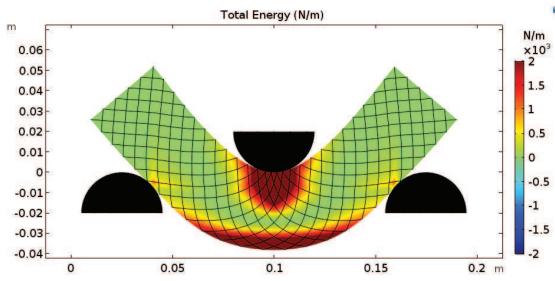

(e) First gradient model $\left(u_{0}=40 \mathrm{~mm}\right)$

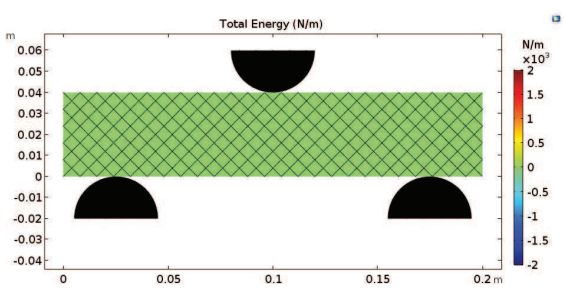

(b) Second gradient model $\left(u_{0}=0 \mathrm{~mm}\right)$.

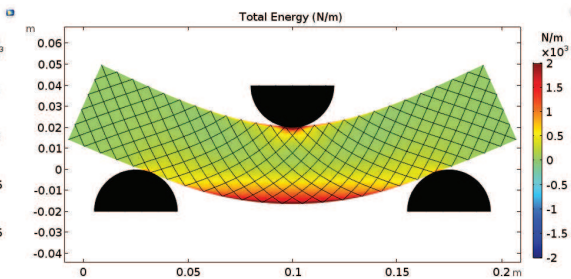

(d) Second gradient model $\left(u_{0}=20 \mathrm{~mm}\right)$.

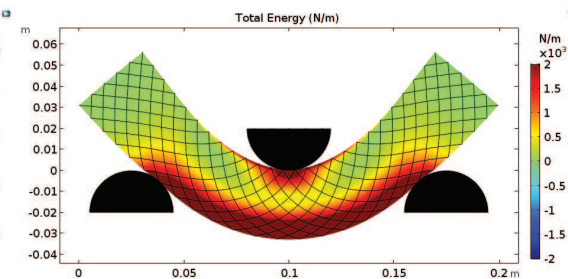

(f) Second gradient model $\left(u_{0}=40 \mathrm{~mm}\right)$.

Figure I2. Comparison between the first gradient and second gradient models.

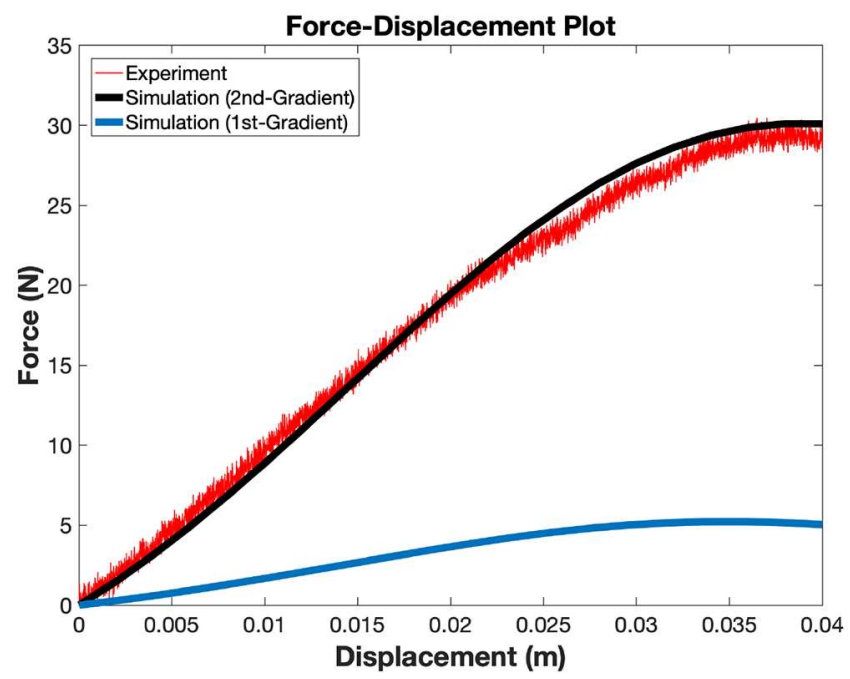

Figure 13. Force-displacement curves (experiment compared with simulation).

result obtained by the second gradient model compare perfectly well those measured by experiments. Also, it must be noticed that the fitted stiffness parameters provide an accurate result in the simulations.

\section{Conclusions and future research perspectives}

In this study, planar deformation of pantographic blocks under a three-point bending test is investigated via a numerical model and experiments. The out-of-plane displacements of pantographic blocks under study are ignored, both in the formulation of the mathematical model and in the measurements performed. However, we obtained a substantial agreement between theoretical predictions and experimental evidence, which is really 
promising. The applicability of the proposed numerical model is shown in the comparisons presented in Figures 9, 10, and 13. Moreover, the contribution of each term in the deformation energy (i.e. stretching, bending, and shearing energies) has been investigated, with the plots provided in Figure 11, and force-displacement plots of both the numerical simulation and the experiment are compared in Figure 13.

It is clear that this circumstance seems to indicate that the formulation of the mathematical model and the implementation of the numerical code are to be taken into account for 3D displacements in future studies. This generalization may present some difficulties, such as well-posedness (see Eremeyev et al. [92]) and postulating a suitable deformation energy for 3D pantographic blocks. Indeed, we have observed that the pantographic block has deformation in the direction orthogonal to the plane of bending, which has some features of anticlastic curvature observed in standard elasticity (e.g. see Love [93]). To investigate these unique behaviors, more complicated experimental setup and apparatus are to be designed, to give an accurate measurement for the deformations of pantographic metamaterials. It would also be quite interesting to conduct the three-point bending test with a longer beam-like block to investigate which kind of dimension reduction beam model would correspond to the present second gradient plane elasticity model.

New theoretical and experimental advancements are needed to confront the 3D deformation patterns that have been observed and involve, at least, some novel Poisson effects. Obviously, a simple superposition of pantographic sheets is not the only way to form 3D blocks with pantographic microstructures. Other design solutions showing very interesting features may also be possible. The mathematical modeling challenges involved in 3D deformation seem rather complex but we are confident that they may be confronted.

\section{Funding}

The author(s) received no financial support for the research, authorship, and/or publication of this article.

\section{ORCID iDs}

M. Erden Yildizdag (iD) https://orcid.org/0000-0003-3041-133X

Emilio Barchiesi (iD https://orcid.org/0000-0002-7296-0671

\section{References}

[1] Kadic, M, Bückmann, T, Stenger, N, et al. On the practicability of pentamode mechanical metamaterials. Appl Phys Lett 2012; 100(19): 049902.

[2] Lee, JH, Singer, JP, and Thomas, EL. Micro-/nanostructured mechanical metamaterials. Adv Mater 2012; 24(36): 4782-4810.

[3] Turco, E, Giorgio, I, Misra, A, et al. King post truss as a motif for internal structure of (meta)material with controlled elastic properties. R Soc Open Sci 2017; 4: 171153.

[4] Vangelatos, Z, Komvopoulos, K, and Grigoropoulos, C. Vacancies for controlling the behavior of microstructured threedimensional mechanical metamaterials. Math Mech Solids 2019; 24(2): 511-524.

[5] Vangelatos, Z, Melissinaki, V, Farsari, M, et al. Intertwined microlattices greatly enhance the performance of mechanical metamaterials. Math Mech Solids 2019; 24(8): 2636-2648.

[6] Vangelatos, Z, Gu, GX, and Grigoropoulos, CP. Architected metamaterials with tailored 3D buckling mechanisms at the microscale. Extreme Mech Lett 2019; 33: 100580.

[7] Eugster, S, dell'Isola, F, and Steigmann, D. Continuum theory for mechanical metamaterials with a cubic lattice substructure. Math Mech Complex Syst 2019; 7(1): 75-98.

[8] Nejadsadeghi, N, Placidi, L, Romeo, M, et al. Frequency band gaps in dielectric granular metamaterials modulated by electric field. Mech Res Commun 2019; 95: 96-103.

[9] Barchiesi, E, Spagnuolo, M, and Placidi, L. Mechanical metamaterials: A state of the art. Math Mech Solids 2019; 24(1): 212234.

[10] Yu, X, Zhou, J, Liang, H, et al. Mechanical metamaterials associated with stiffness, rigidity and compressibility: A brief review. Prog Mater Sci 2018; 94: 114-173.

[11] dell'Isola, F, Seppecher, P, Alibert, JJ, et al. Pantographic metamaterials: An example of mathematically driven design and of its technological challenges. Continuum Mech Thermodyn 2019; 31(4): 851-884.

[12] dell'Isola, F, Seppecher, P, Spagnuolo, M, et al. Advances in pantographic structures: Design, manufacturing, models, experiments and image analyses. Continuum Mech Thermodyn 2019; 31(4): 1231-1282.

[13] Alibert, JJ, Seppecher, P, and dell'Isola, F. Truss modular beams with deformation energy depending on higher displacement gradients. Math Mech Solids 2003; 8(1): 51-73. 
[14] dell'Isola, F, Andreaus, U, Cazzani, A, et al. The complete works of Gabrio Piola: Volume II commented English translation. Cham: Springer, 2018.

[15] dell'Isola, F, Andreaus, U, and Placidi, L. At the origins and in the vanguard of peridynamics, non-local and higher-gradient continuum mechanics: An underestimated and still topical contribution of Gabrio Piola. Math Mech Solids 2015; 20(8): 887-928.

[16] dell'Isola, F, Della Corte, A, Esposito, R, et al. Some cases of unrecognized transmission of scientific knowledge: From antiquity to Gabrio Piola's peridynamics and generalized continuum theories. In: Altenbach, H and Forest, S (eds.) Generalized continua as models for classical and advanced materials (Advanced Structured Materials, vol. 42). Cham: Springer, $2016,77-128$.

[17] Auffray, N, dell'Isola, F, Eremeyev, VA, et al. Analytical continuum mechanics à la Hamilton-Piola least action principle for second gradient continua and capillary fluids. Math Mech Solids 2015; 20(4): 375-417.

[18] Rahali, Y, Giorgio, I, Ganghoffer, J, et al. Homogenization à la Piola produces second gradient continuum models for linear pantographic lattices. Int J Eng Sci 2015; 97: 148-172.

[19] Pideri, C, and Seppecher, P. A second gradient material resulting from the homogenization of an heterogeneous linear elastic medium. Continuum Mech Thermodyn 1997; 9(5): 241-257.

[20] Abdoul-Anziz, H, and Seppecher, P. Strain gradient and generalized continua obtained by homogenizing frame lattices. Math Mech Complex Syst 2018; 6(3): 213-250.

[21] Abdoul-Anziz, H, Seppecher, P, and Bellis, C. Homogenization of frame lattices leading to second gradient models coupling classical strain and strain-gradient terms. Math Mech Solids 2019; 24(12): 3976-3999.

[22] dell'Isola, F, Giorgio, I, Pawlikowski, M, et al. Large deformations of planar extensible beams and pantographic lattices: Heuristic homogenization, experimental and numerical examples of equilibrium. Proc R Soc London, Ser A 2016; 472(2185): 20150790.

[23] Cuomo, M, dell'Isola, F, and Greco, L. Simplified analysis of a generalized bias test for fabrics with two families of inextensible fibres. Z Angew Math Phys 2016; 67(3): 61.

[24] dell'Isola, F, Cuomo, M, Greco, L, et al. Bias extension test for pantographic sheets: Numerical simulations based on second gradient shear energies. J Eng Math 2017; 103(1): 127-157.

[25] Placidi, L, Greco, L, Bucci, S, et al. A second gradient formulation for a 2D fabric sheet with inextensible fibres. $Z$ Angew Math Phys 2016; 67(5): 114 .

[26] Spagnuolo, M, Barcz, K, Pfaff, A, et al. Qualitative pivot damage analysis in aluminum printed pantographic sheets: Numerics and experiments. Mech Res Commun 2017; 83: 47-52.

[27] Andreaus, U, Spagnuolo, M, Lekszycki, T, et al. A Ritz approach for the static analysis of planar pantographic structures modeled with nonlinear Euler-Bernoulli beams. Continuum Mech Thermodyn 2018; 30(5): 1103-1123.

[28] De Angelo, M, Spagnuolo, M, D'Annibale, F, et al. The macroscopic behavior of pantographic sheets depends mainly on their microstructure: Experimental evidence and qualitative analysis of damage in metallic specimens. Continuum Mech Thermodyn 2019; 31: 1181-1203.

[29] Giorgio, I. Numerical identification procedure between a micro-Cauchy model and a macro-second gradient model for planar pantographic structures. Z Angew Math Phys 2016; 67(4): 95.

[30] Placidi, L, Andreaus, U, and Giorgio, I. Identification of two-dimensional pantographic structure via a linear D4 orthotropic second gradient elastic model. J Eng Math 2017; 103(1): 1-21.

[31] Yang, H, Ganzosch, G, Giorgio, I, et al. Material characterization and computations of a polymeric metamaterial with a pantographic substructure. Z Angew Math Phys 2018; 69(4): 105.

[32] De Angelo, M, Barchiesi, E, Giorgio, I, et al. Numerical identification of constitutive parameters in reduced-order bi-dimensional models for pantographic structures: Application to out-of-plane buckling. Arch Appl Mech 2019; 89(7): 1333-1358.

[33] Nejadsadeghi, N, De Angelo, M, Drobnicki, R, et al. Parametric experimentation on pantographic unit cells reveals local extremum configuration. Exp Mech 2019; 59(6): 927-939.

[34] Solyaev, Y, Lurie, S, Barchiesi, E, et al. On the dependence of standard and gradient elastic material constants on a field of defects. Math Mech Solids 2020; 25(1): 35-45.

[35] Turco, E. How the properties of pantographic elementary lattices determine the properties of pantographic metamaterials. In: Abali, B, Altenbach, H, dell'Isola, F, et al. (eds.) New achievements in continuum mechanics and thermodynamics (Advanced Structured Materials, vol. 108). Cham: Springer, 2019, 489-506.

[36] Steigmann, DJ, and dell'Isola, F. Mechanical response of fabric sheets to three-dimensional bending, twisting, and stretching. Acta Mech Sin 2015; 31(3): 373-382.

[37] Giorgio, I, Della Corte, A, dell'Isola, F, et al. Buckling modes in pantographic lattices. CR Mec 2016; 344(7): 487-501.

[38] Giorgio, I, Rizzi, NL, and Turco, E. Continuum modelling of pantographic sheets for out-of-plane bifurcation and vibrational analysis. Proc R Soc London, Ser A 2017; 473(2207): 20170636.

[39] Scerrato, D, Giorgio, I, and Rizzi, NL. Three-dimensional instabilities of pantographic sheets with parabolic lattices: Numerical investigations. Z Angew Math Phys 2016; 67(3): 53.

[40] Niiranen, J, and Niemi, AH. Variational formulations and general boundary conditions for sixth-order boundary value problems of gradient-elastic Kirchhoff plates. Eur J Mech A Solids 2017; 61: 164-179.

[41] Khakalo, S, Balobanov, V, and Niiranen, J. Modelling size-dependent bending, buckling and vibrations of 2D triangular lattices by strain gradient elasticity models: Applications to sandwich beams and auxetics. Int J Eng Sci 2018; 127: 33-52.

[42] Khakalo, S, and Niiranen, J. Lattice structures as thermoelastic strain gradient metamaterials: Evidence from full-field simulations and applications to functionally step-wise-graded beams. Composites Part B 2019; 177: 107224. 
[43] Barchiesi, E, and Khakalo, S. Variational asymptotic homogenization of beam-like square lattice structures. Math Mech Solids 2019; 24(10): 3295-3318.

[44] Eremeyev, V. Nonlinear micropolar shells: Theory and applications. In: Pietraszkiewicz, W and Szymczak, C (eds.) Shell Structures: Theory and Applications. London: Taylor \& Francis, 2005, 11-18.

[45] Eremeyev, VA, Lebedev, LP, and Altenbach, H. Foundations of micropolar mechanics. Berlin: Springer Science \& Business Media, 2012.

[46] Eremeyev, V, and Altenbach, H. Basics of mechanics of micropolar shells. In: Altenbach, H and Eremeyev, V (eds.) Shell-like structures. Cham: Springer, 2017, 63-111.

[47] Altenbach, H, and Eremeyev, VA. On the theories of plates based on the Cosserat approach. In: Maugin, G and Metrikine, A (eds.) Mechanics of generalized continua (Advances in Mechanics and Mathematics, vol 21). New York: Springer, $2010,27-35$.

[48] Altenbach, H, and Eremeyev, VA. On the linear theory of micropolar plates. J Appl Math Mech 2009; 89(4): $242-256$.

[49] Altenbach, H, and Eremeyev, VA. Cosserat-type shells. In: Altenbach, H and Eremeyev, VA (eds.) Generalized continua from the theory to engineering applications (CISM International Centre for Mechanical Sciences (Courses and Lectures), vol. 541). Vienna: Springer, 2013, 131-178.

[50] Altenbach, H, Eremeyev, VA, and Lebedev, LP. Micropolar shells as two-dimensional generalized continua models. In: Altenbach, H, Maugin, G, and Erofeev, V (eds.) Mechanics of generalized continua (Advanced Structured Materials, vol. 7). Berlin: Springer, 2011, 23-55.

[51] Misra, A and Poorsolhjouy, P. Identification of higher-order elastic constants for grain assemblies based upon granular micromechanics. Math Mech Complex Syst 2015; 3(3): 285-308.

[52] Placidi, L, Barchiesi, E, Turco, E, et al. A review on 2D models for the description of pantographic fabrics. Z Angew Math Phys 2016; 67(5): 121.

[53] Turco, E, Golaszewski, M, Cazzani, A, et al. Large deformations induced in planar pantographic sheets by loads applied on fibers: Experimental validation of a discrete Lagrangian model. Mech Res Commun 2016; 76: 51-56.

[54] Turco, E, Barcz, K, Pawlikowski, M, et al. Non-standard coupled extensional and bending bias tests for planar pantographic lattices. Part I: Numerical simulations. Z Angew Math Phys 2016; 67(5): 122.

[55] Turco, E, Golaszewski, M, Giorgio, I, et al. Pantographic lattices with non-orthogonal fibres: Experiments and their numerical simulations. Composites Part B 2017; 118: 1-14.

[56] Turco, E, Misra, A, Sarikaya, R, et al. Quantitative analysis of deformation mechanisms in pantographic substructures: Experiments and modeling. Continuum Mech Thermodyn 2019; 31(1): 209-223.

[57] Battista, A, Rosa, L, dell'Erba, R, et al. Numerical investigation of a particle system compared with first and second gradient continua: Deformation and fracture phenomena. Math Mech Solids 2017; 22(11): 2120-2134.

[58] Greco, L, Giorgio, I, and Battista, A. In plane shear and bending for first gradient inextensible pantographic sheets: Numerical study of deformed shapes and global constraint reactions. Math Mech Solids 2017; 22(10): 1950-1975.

[59] Franciosi, P, Spagnuolo, M, and Salman, OU. Mean Green operators of deformable fiber networks embedded in a compliant matrix and property estimates. Continuum Mech Thermodyn 2019; 31(1): 101-132.

[60] Chiaia, B, Barchiesi, E, De Biagi, V, et al. A novel worst-case-based structural resilience index: Definition, computation and applications to portal frame structures. Mech Res Commun 2019; 99: 52-57.

[61] Barchiesi, E, Eugster, SR, Placidi, L, et al. Pantographic beam: A complete second gradient 1D-continuum in plane. $Z$ Angew Math Phys 2019; 70(5): 135.

[62] Spagnuolo, M, and Andreaus, U. A targeted review on large deformations of planar elastic beams: Extensibility, distributed loads, buckling and post-buckling. Math Mech Solids 2019; 24(1): 258-280.

[63] Green, A. Micro-materials and multipolar continuum mechanics. Int J Eng Sc 1965; 3(5): 533-537.

[64] Mindlin, RD. Second gradient of strain and surface-tension in linear elasticity. Int J Solids Struct 1965; 1(4): 417-438.

[65] Sedov, LI. Mathematical methods for constructing new models of continuous media. Russ Math Surv 1965; 20 (5): 123.

[66] Toupin, RA. Theories of elasticity with couple-stress. Arch Ration Mech Anal 1964; 17(2): 85-112.

[67] Germain, P. The method of virtual power in continuum mechanics. Part 2: Microstructure. SIAM J Appl Math 1973; 25(3): $556-575$.

[68] Turco, E, and Rizzi, NL. Pantographic structures presenting statistically distributed defects: Numerical investigations of the effects on deformation fields. Mech Res Commun 2016; 77: 65-69.

[69] Misra, A, Lekszycki, T, Giorgio, I, et al. Pantographic metamaterials show atypical Poynting effect reversal. Mech Res Commun 2018; 89: 6-10.

[70] Javanbakht, M, and Barati, E. Martensitic phase transformations in shape memory alloy: Phase field modeling with surface tension effect. Comput Mater Sci 2016; 115: 137-144.

[71] Mirzakhani, S, and Javanbakht, M. Phase field-elasticity analysis of austenite-martensite phase transformation at the nanoscale: Finite element modeling. Comput Mater Sci 2018; 154: 41-52.

[72] dell'Isola, F, Lekszycki, T, Pawlikowski, M, et al. Designing a light fabric metamaterial being highly macroscopically tough under directional extension: First experimental evidence. Z Angew Math Phys 2015; 66(6): 3473-3498.

[73] della Corte, A, Giorgio, I, and Scerrato, D. Pantographic 2D sheets: Discussion of some numerical investigations and potential applications. Int J Non Linear Mech 2016; 80: 200-208.

[74] Fischer, P, Klassen, M, Mergheim, J, et al. Isogeometric analysis of 2D gradient elasticity. Comput Mech 2011; 47(3): 325-334. 
[75] Cuomo, M, Contrafatto, L, and Greco, L. A variational model based on isogeometric interpolation for the analysis of cracked bodies. Int J Eng Sci 2014; 80: 173-188.

[76] Cazzani, A, Malagù, M, and Turco, E. Isogeometric analysis: A powerful numerical tool for the elastic analysis of historical masonry arches. Continuum Mech Thermodyn 2016; 28(1-2): 139-156.

[77] Cazzani, A, Malagù, M, Turco, E, et al. Constitutive models for strongly curved beams in the frame of isogeometric analysis. Math Mech Solids 2016; 21(2): 182-209.

[78] Cazzani, A, Malagù, M, and Turco, E. Isogeometric analysis of plane-curved beams. Math Mech Solids 2016; 21 (5): $562-577$.

[79] Cazzani, A, Stochino, F, and Turco, E. An analytical assessment of finite element and isogeometric analyses of the whole spectrum of Timoshenko beams. J Appl Math Mech 2016; 96(10): 1220-1244.

[80] Niiranen, J, Khakalo, S, Balobanov, V, et al. Variational formulation and isogeometric analysis for fourth-order boundary value problems of gradient-elastic bar and plane strain/stress problems. Comput Methods Appl Mech Eng 2016; 308: $182-211$.

[81] Niiranen, J, Kiendl, J, Niemi, AH, et al. Isogeometric analysis for sixth-order boundary value problems of gradient-elastic Kirchhoff plates. Comput Methods Appl Mech Eng 2017; 316: 328-348.

[82] Niiranen, J, Balobanov, V, Kiendl, J, et al. Variational formulations, model comparisons and numerical methods for EulerBernoulli micro-and nano-beam models. Math Mech Solids 2019; 24(1): 312-335.

[83] Balobanov, V, and Niiranen, J. Locking-free variational formulations and isogeometric analysis for the Timoshenko beam models of strain gradient and classical elasticity. Comput Methods Appl Mech Eng 2018; 339: 137-159.

[84] Khakalo, S, and Niiranen, J. Isogeometric analysis of higher-order gradient elasticity by user elements of a commercial finite element software. Comput-Aided Des 2017; 82: 154-169.

[85] Khakalo, S, and Niiranen, J. Anisotropic strain gradient thermoelasticity for cellular structures: Plate models, homogenization and isogeometric analysis. J Mech Phys Solids 2020; 134: 103728.

[86] Yaghoubi, ST, Balobanov, V, Mousavi, SM, et al. Variational formulations and isogeometric analysis for the dynamics of anisotropic gradient-elastic Euler-Bernoulli and shear-deformable beams. Eur J Mech A Solids 2018; 69: 113-123.

[87] Yildizdag, ME, Demirtas, M, and Ergin, A. Multipatch discontinuous Galerkin isogeometric analysis of composite laminates. Continuum Mech Thermodyn Epub ahead of print 19 July 2018. DOI: 10.1007/s00161-018-0696-9.

[88] Yildizdag, ME, Ardic, IT, Demirtas, M, et al. Hydroelastic vibration analysis of plates partially submerged in fluid with an isogeometric FE-BE approach. Ocean Eng 2019; 172: 316-329.

[89] Capobianco, G, Eugster, SR, and Winandy, T. Modeling planar pantographic sheets using a nonlinear Euler-Bernoulli beam element based on B-spline functions. Proc Appl Math Mech 2018; 18: e201800220.

[90] Greco, L, Cuomo, M, and Contrafatto, L. A reconstructed local $\bar{B}$ formulation for isogeometric Kirchhoff-Love shells. Comput Methods Appl Mech Eng 2018; 332: 462-487.

[91] Balobanov, V, Kiendl, J, Khakalo, S, et al. Kirchhoff-Love shells within strain gradient elasticity: Weak and strong formulations and an H3-conforming isogeometric implementation. Comput Methods Appl Mech Eng 2019; 344: 837-857.

[92] Eremeyev, VA, dell'Isola, F, Boutin, C, et al. Linear pantographic sheets: Existence and uniqueness of weak solutions. $J$ Elast 2018; 132(2): 175-196.

[93] Love, AEH. A treatise on the mathematical theory of elasticity. Cambridge: Cambridge University Press, 2013. 